\title{
A PREFERRED APPROACH TO THE LINEARIZATION OF TURBULENT BOUNDARY-LAYER EQUATIONS
}

\author{
Hong-Ming JANG ${ }^{2}$, Tuncer Cebeci ${ }^{1}$ and Herbert B. Keller ${ }^{3}$ \\ 'Aerospace Engineering Department, California State University, Long Beach, CA 90840, U.S.A., \\ ${ }^{2}$ Mechanical Engineering Department, University of Michigan, Ann Arbor, Mich, U.S.A. and \\ ${ }^{3}$ Department of Applied Mathematics, California Institute of Technology, Pasadena, Calif, U.S.A.
}

(Received 28 September 1988)

\begin{abstract}
An efficient procedure for solving the fully linearized form of the boundary-layer equations is described for turbulent flows. The procedure makes use of the so-called bordering algorithm and is applicable to problems in which the structure of the linearized system of equations deviates from the block triagonal matrix form which may be caused by boundary conditions.
\end{abstract}

\section{INTRODUCTION}

The solution of the two-dimensional boundary-layer equations for a laminar flow involves linearization of all terms by Newton's method and can lead to a set of three first-order equations [1]. The same approach to the corresponding turbulent form of the boundary-layer equations, with an eddy-viscosity representation of the turbulent diffusion term, leads to incomplete linearization as in Ref. [1] or to complete linearization with five first-order equations as in Ref. [2]. Important consequences of the former approach are slow convergence, with increased computing times and on occasion, an inability to converge to a specified criterion. While the approach of Ref. [2] provides quadratic convergence of the solutions as in laminar flows, the need to increase the system of equations from 3 to 5 makes it difficult to apply to the more complex problems, and particularly those involving three independent variables. The algorithm described and applied in this paper, is designed to achieve complete linearization of the turbulent boundary-layer equations, without increase in the number of first-order equations, and greater generality of application.

The present method is based on the bordering algorithm of Keller [3,4], and is applied to the boundary-layer equations of Section 2 with the eddy-viscosity formulation of Cebeci and Smith [1]. The finite-difference representation of the equations is described briefly in Section 3, and the present form of the bordering algorithm in Section 4. Sample results are presented in Section 5 .

\section{BASIC EQUATIONS}

The boundary-layer equations and their boundary conditions for 2-D incompressible laminar and turbulent flows are well known and, with the concept of eddy viscosity, $\epsilon_{\mathrm{m}}$, and introducing $b=1+\epsilon_{\mathrm{m}} / v$, they can be written as:

$$
\begin{gathered}
\frac{\partial u}{\partial x}+\frac{\partial v}{\partial y}=0 \\
u \frac{\partial u}{\partial x}+v \frac{\partial u}{\partial y}=u_{\mathrm{e}} \frac{\mathrm{d} u_{\mathrm{e}}}{\mathrm{d} x}+v \frac{\partial}{\partial y}\left(b \frac{\partial u}{\partial y}\right) \\
y=0, \quad u=v=0 ; \quad y \rightarrow \delta, \quad u=u_{\mathrm{e}} .
\end{gathered}
$$

The presence of the eddy viscosity $\epsilon_{\mathrm{m}}$ in $b$ requires a turbulence model, and the algebraic eddy-viscosity formulation of Cebeci and Smith [1] is used here. According to this formulation, $\epsilon_{m}$ 
is defined by two separate formulas given by:

$$
\epsilon_{\mathrm{m}}= \begin{cases}\left\{0.4 y\left[1-\exp \left(\frac{-y}{A}\right)\right]\right\}^{2} \frac{\partial u}{\partial y} & 0 \leqslant y \leqslant y_{\mathrm{c}} \\ 0.0168 \int_{0}^{\infty}\left(u_{\mathrm{e}}-u\right) \mathrm{d} y & y_{\mathrm{c}} \leqslant y \leqslant \delta\end{cases}
$$

where:

$$
A=26 v u_{\tau}^{-1} N, \quad N=\left(1-11.8 p^{+}\right)^{-1 / 2}, \quad u_{\tau}=\left(\frac{\tau_{\mathrm{w}}}{\rho}\right)^{1 / 2}, \quad p^{+}=\frac{v u_{\mathrm{e}}}{u_{\tau}^{3}} \frac{\mathrm{d} u_{\mathrm{e}}}{\mathrm{d} x} .
$$

The condition used in the determination of $y_{\mathrm{c}}$ is the continuity of the eddy viscosity so that eqn (4a) is applied from the wall outwards until its value is equal to that given for the outer region by eqn (4b).

For external flows, it is convenient to solve (1) and (2) in transformed variables, and the Falkner-Skan transformation [1] is used for this purpose. The result, with $m=\left(x / u_{\mathrm{e}}\right) \mathrm{d} u_{\mathrm{e}} / \mathrm{d} x$ and with primes denoting differentiation with respect to the similarity variable $\eta$, is:

$$
\begin{gathered}
\left(b f^{\prime \prime}\right)^{\prime}+\frac{m+1}{2} f f^{\prime \prime}+m\left[1-\left(f^{\prime}\right)^{2}\right]=x\left(f^{\prime} \frac{\partial f^{\prime}}{\partial x}-f^{\prime \prime} \frac{\partial f}{\partial x}\right), \\
\eta=0, \quad f^{\prime}=f=0 ; \quad \eta \rightarrow \eta_{e}, \quad f^{\prime} \rightarrow 1 .
\end{gathered}
$$

In terms of transformed variables, the $b$ term in eqn (6) can be written as:

$$
b=1+a_{1} f^{\prime \prime}\left[1-\exp \left(-a_{2} N^{-1} f_{\mathrm{w}}^{\prime \prime \prime / 2}\right)\right]^{2} \lambda_{1}+a_{3}\left(\eta_{\mathrm{e}}-f_{\mathrm{e}}\right) \lambda_{2},
$$

where $\lambda_{1}$ and $\lambda_{2}$ are determined by the continuity of the eddy-viscosity formulas with $\lambda_{1}=1$ and $\lambda_{2}=0$ in the inner region and $\lambda_{1}=0$ and $\lambda_{2}=1$ in the outer region. To ensure a smoother result, values of $\lambda_{1}=\lambda_{2}=1 / 2$ were used near the interface of both regions. The terms $a_{1}, a_{2}, a_{3}$, and $N$ are defined by:

$$
\begin{gathered}
a_{1}=0.16 R_{x}^{1 / 2} \eta^{2}, \quad a_{2}=\left(R_{x}^{1 / 4} / 26\right) \eta, \quad a_{3}=0.0168 R_{x}^{1 / 2} \\
a_{4}=11.8 m R_{r}^{-1 / 4}, \quad N=\left[1-a_{4}\left(f_{\mathrm{w}}^{\prime \prime}\right)^{-3 / 2}\right]^{-1 / 2}, \quad R_{x}=u_{\mathrm{e}} x / v
\end{gathered}
$$

\section{SOLUTION PROCEDURE}

The solution of eqns (6) and (7) is obtained by Keller's box method [1,5] for which the new variables $u$ and $v$, defined by:

$$
f^{\prime}=u, \quad u^{\prime}=v,
$$

allow eqn (6) to be written as the first-order system (10a) and:

$$
(b v)^{\prime}+\frac{m+1}{2} f v+m\left(1-u^{2}\right)=x\left(u \frac{\partial u}{\partial x}-v \frac{\partial f}{\partial x}\right) .
$$

Next, on a finite-difference net denoted by:

$$
\begin{gathered}
x_{1}=0, \quad x_{n}=x_{n-1}+k_{n-1}, \quad n=2, \ldots, N \\
\eta_{1}=0, \quad \eta_{j}=\eta_{j-1}+h_{j-1}, \quad j=2, \ldots, J ; \quad \eta_{J}=\eta_{\mathrm{e}}
\end{gathered}
$$

we approximate the quantities $(f, u, v)$ at points $\left(x_{n}, \eta_{j}\right)$ by $\left(f_{j}^{n}, u_{j}^{n}, v_{j}^{n}\right)$. The finite-difference approximations for eqn (10a) are then written using centered difference quotients and averaged about the midpoint $\left(x_{n}, \eta_{j-1 / 2}\right)$ of a mesh interval and those for eqn $(10 \mathrm{~b})$ are written at the midpoint $\left(x_{n-1 / 2}, \eta_{j-1 / 2}\right)$ of a mesh rectangle to obtain:

$$
\begin{gathered}
f_{j}^{n}-f_{j-1}^{n}-\left(h_{j-1} / 2\right)\left(u_{j}^{n}+u_{j-1}^{n}\right)=0 \\
u_{j}^{n}-u_{j-1}^{n}-\left(h_{j-1} / 2\right)\left(v_{j}^{n}+v_{j-1}^{n}\right)=0 \\
h_{j-1}^{-1}\left[(b v)_{j}^{n}-(b v)_{j-1}^{n}\right]+\alpha_{1}(f v)_{j-1 / 2}^{n}-\alpha_{2}\left(u^{2}\right)_{j-1 / 2}^{n}+\alpha\left(v_{j-1 / 2}^{n-1} f_{j-1 / 2}^{n}-f_{j-1 / 2}^{n-1} v_{j-1 / 2}^{n}\right)=R_{j-1 / 2}^{n-1},
\end{gathered}
$$


where:

$$
\begin{aligned}
R_{j-1 / 2}^{n-1} & =-L_{j-1 / 2}^{n-1}+\alpha\left[(f v)_{j-1 / 2}^{n-1}-\left(u^{2}\right)_{j-1 / 2}^{n-1}\right]-m^{n} \\
L_{j-1 / 2}^{n-1} & =\left\{h_{j-1}^{-1}\left(b_{j} v_{j}-b_{j-1} v_{j-1}\right)+m_{1}(f v)_{j-1 / 2}+m\left[1-\left(u^{2}\right)_{j-1 / 2}\right]\right\}^{n-1} \\
\alpha_{1} & =\alpha+m_{1}, \quad \alpha_{2}=\alpha+m, \quad \alpha=\left(x_{n-1 / 2} / k_{n-1}\right), \quad m_{1}=(m+1) / 2 .
\end{aligned}
$$

With the application of Newton's method, the linearized equations and boundary conditions become at the advanced $x$-location, $x=x_{n}$, on suppression of the subscript $n$ :

$$
\begin{gathered}
\delta f_{j}-\delta f_{j-1}-\frac{h_{j-1}}{2}\left(\delta u_{j}+\delta u_{j-1}\right)=\left(r_{1}\right)_{j} \\
\delta u_{j}-\delta u_{j-1}-\frac{h_{j-1}}{2}\left(\delta v_{j}+\delta v_{j-1}\right)=\left(r_{3}\right)_{j-1}
\end{gathered}
$$

$\left(S_{8}\right)_{j} \delta v_{1}+\left(S_{1}\right)_{j} \delta v_{j}+\left(S_{2}\right)_{j} \delta v_{j-1}+\left(S_{3}\right)_{j} \delta f_{j}+\left(S_{4}\right)_{j} \delta f_{j-1}+\left(S_{5}\right)_{j} \delta u_{j}$

$$
+\left(S_{6}\right)_{j} \delta u_{j-1}+\left(S_{7}\right)_{j} \delta f_{J}=\left(r_{2}\right)_{j}
$$

$$
\delta f_{1}=0, \quad \delta u_{1}=0 \quad \text { and } \delta u_{J}=0,
$$

where the coefficients $\left(s_{k}\right)_{j}(k=1-8)$ and $\left(r_{s}\right)_{j}(s=1-3)$ are given in Ref. [6].

Introducing appropriate vectors and matrices the linear system given by eqns (13) and (14) can be written in the following matrix-vector form:

$$
\mathbf{A} \boldsymbol{\delta}=\mathbf{r}
$$

where

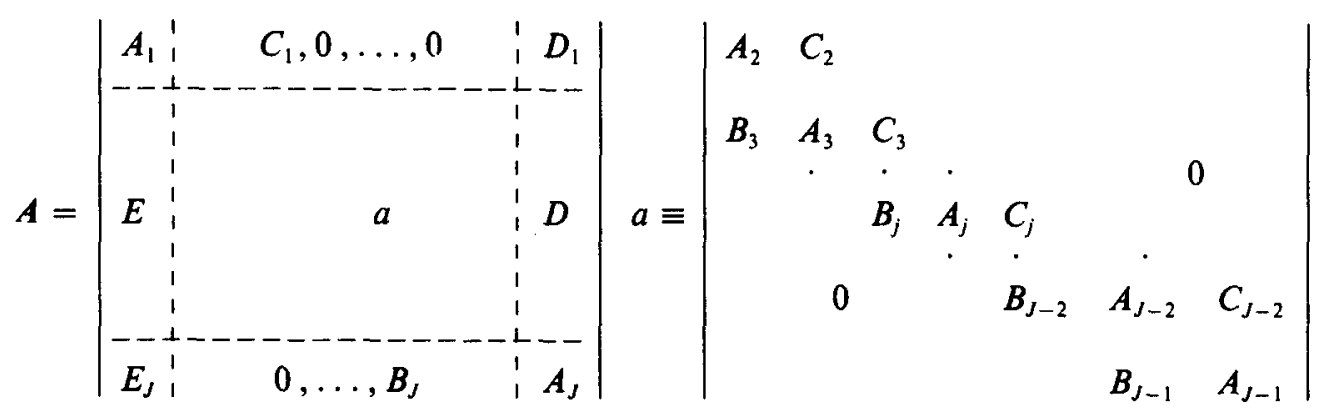

$$
\begin{gathered}
\boldsymbol{\delta} \equiv\left|\begin{array}{l}
\boldsymbol{\delta}_{1} \\
\boldsymbol{\Delta} \\
\boldsymbol{\delta}_{J}
\end{array}\right|, \quad \Delta \equiv\left|\begin{array}{c}
\boldsymbol{\delta}_{2} \\
\vdots \\
\boldsymbol{\delta}_{j} \\
\vdots \\
\boldsymbol{\delta}_{J-1}
\end{array}\right|, \quad \boldsymbol{\delta}_{j} \equiv\left|\begin{array}{c}
\delta f_{j} \\
\delta u_{j} \\
\delta v_{j}
\end{array}\right| \quad 1 \leqslant j \leqslant J \\
\mathbf{r} \equiv\left|\begin{array}{l}
\mathbf{r}_{1} \\
\mathbf{R} \\
\mathbf{r}_{J}
\end{array}\right|, \quad \mathbf{r}_{1} \equiv\left|\begin{array}{c}
0 \\
0 \\
\left(r_{3}\right)_{1}
\end{array}\right|, \quad \mathbf{R} \equiv\left|\begin{array}{c}
\mathbf{r}_{2} \\
\vdots \\
\mathbf{r}_{j} \\
\vdots \\
\mathbf{r}_{J-1}
\end{array}\right| \\
\mathbf{r}_{j} \equiv\left|\begin{array}{l}
\left(r_{1}\right)_{j} \\
\left(r_{2}\right)_{j} \\
\left(r_{3}\right)_{j}
\end{array}\right| \quad 2 \leqslant j \leqslant J-1, \quad \mathbf{r}_{J} \equiv\left|\begin{array}{c}
\left(r_{1}\right)_{J} \\
\left(r_{2}\right)_{J} \\
0
\end{array}\right|
\end{gathered}
$$


Here each of $A_{j}, B_{j}, C_{j}, D_{j}, E_{j}$ is a $3 \times 3$ matrix given by:

$$
\begin{aligned}
& A_{1} \equiv\left|\begin{array}{rrr}
1 & 0 & 0 \\
0 & 1 & 0 \\
0 & -1 & -\frac{h_{1}}{2}
\end{array}\right|, \quad A_{j} \equiv\left|\begin{array}{ccc}
1 & -\frac{h_{j-1}}{2} & 0 \\
\left(S_{3}\right)_{j} & \left(S_{5}\right)_{j} & \left(S_{1}\right)_{j} \\
0 & -1 & -\frac{h_{j}}{2}
\end{array}\right| 2 \leqslant j \leqslant j-1 \\
& A_{J} \equiv\left|\begin{array}{ccc}
1 & -\frac{h_{J-1}}{2} & 0 \\
\left(S_{3}\right)_{J}+\left(S_{7}\right)_{J} & \left(S_{5}\right)_{J} & \left(S_{1}\right)_{J} \\
0 & 1 & 0
\end{array}\right| \\
& B_{j} \equiv\left|\begin{array}{ccc}
-1 & -\frac{h_{j-1}}{2} & 0 \\
\left(S_{4}\right)_{j} & \left(S_{6}\right)_{j} & \left(S_{2}\right)_{j} \\
0 & 0 & 0
\end{array}\right| 3 \leqslant j \leqslant J, \quad C_{j} \equiv\left|\begin{array}{ccc}
0 & 0 & 0 \\
0 & 0 & 0 \\
0 & 1 & -\frac{h_{j}}{2}
\end{array}\right| 1 \leqslant j \leqslant J-2 \\
& D_{1} \equiv\left|\begin{array}{lll}
0 & 0 & 0 \\
0 & 0 & 0 \\
0 & 0 & 0
\end{array}\right|, \quad D_{j} \equiv\left|\begin{array}{ccc}
0 & 0 & 0 \\
\left(S_{7}\right)_{j} & 0 & 0 \\
0 & 0 & 0
\end{array}\right| \quad 2<j<J-2 \\
& D_{J-1} \equiv\left|\begin{array}{ccc}
0 & 0 & 0 \\
\left(S_{7}\right)_{J-1} & 0 & 0 \\
0 & 1 & -\frac{h_{J-1}}{2}
\end{array}\right| \\
& E_{2} \equiv\left|\begin{array}{ccc}
-1 & -\frac{h_{1}}{2} & 0 \\
\left(S_{4}\right)_{2} & \left(S_{6}\right)_{2} & \left(S_{8}\right)_{2}+\left(S_{2}\right)_{2} \\
0 & 0 & 0
\end{array}\right|, \quad E_{j} \equiv\left|\begin{array}{ccc}
0 & 0 & 0 \\
0 & 0 & \left(S_{8}\right)_{j} \\
0 & 0 & 0
\end{array}\right| 3 \leqslant j \leqslant J
\end{aligned}
$$

Note that the first two rows of $A_{1}, C_{1}, D_{1}$ and $\mathbf{r}_{1}$ and the last rows of $E_{J}, B_{J}, A_{J}$ and $r_{J}$ correspond to the boundary conditions.

For convenience, the $D$ and $E$ matrices can also be written as:

$$
\begin{gathered}
D=\left(\mathbf{D}_{1}, \mathbf{D}_{2}, \mathbf{D}_{3}\right) \\
E=\left(\mathbf{E}_{1}, \mathbf{E}_{2}, \mathbf{E}_{3}\right)
\end{gathered}
$$


where

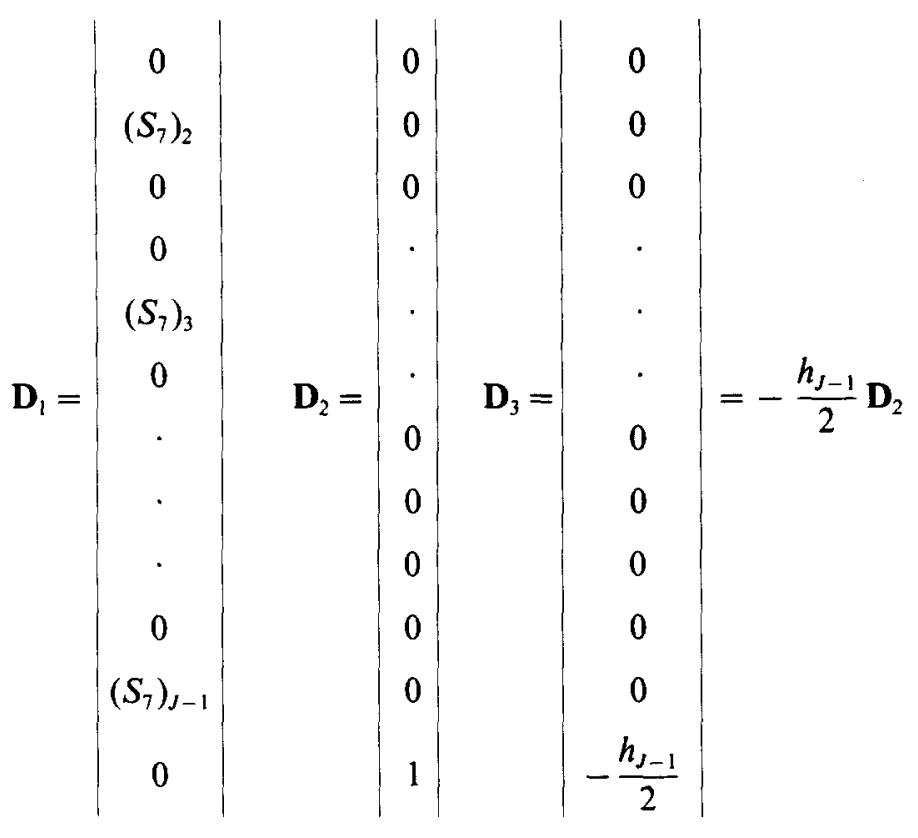

$$
\mathbf{E}_{1}=\left|\begin{array}{c|c}
-1 \\
\left(S_{4}\right)_{2} \\
0 \\
0 \\
0 \\
0 \\
. \\
. \\
0 \\
0 \\
0
\end{array}\right| \mathbf{E}_{2}=\left|\begin{array}{c}
-h_{1} / 2 \\
\left(S_{6}\right)_{2} \\
0 \\
0 \\
0 \\
0 \\
. \\
. \\
. \\
0 \\
0 \\
0
\end{array}\right| \mathbf{E}_{3}=\left|\begin{array}{c}
0 \\
\left(S_{8}\right)_{2}+\left(S_{2}\right)_{2} \\
0 \\
0 \\
\left(S_{8}\right)_{3} \\
0 \\
. \\
. \\
0 \\
\left(S_{8}\right)_{J-1} \\
0
\end{array}\right|
$$

It is useful to compare the coefficient matrix of eqn (15) with that which stems from the approach in which the turbulent diffusion term is not linearized. Equation (15) applies in both cases but here the linearization of the diffusion term leads to additional matrices $D_{j}$ and $E_{j}$, which make the coefficient matrix $A$ differ from that without linearization. In the absence of $D_{j}$ and $E_{j}$, eqn (15) can be solved by the block elimination method described in Ref. [1] but their presence requires a different solution algorithm which is described in the following section.

\section{FULL BORDERING ALGORITHM}

The need to overcome problems such as that described above was recognized by Keller $[3,4]$ who devised an efficient algorithm which involved additional arithmetic operations so that the resulting system could be expressed in the tridiagonal matrix form required by the block-elimination method. Problems in which $E_{j}$ or $D_{j}$ are absent were formulated by an algorithm referred to as half-bordering and those in which both were present made use of the full bordering algorithm. It is possible by reordering the equations and the variables to transform the full bordering case to a half bordering 
case. We do not do that here as the codes and algorithms are simpler in the untransformed variables.

The full-bordering algorithm used here writes eqn (15) as:

$$
\begin{aligned}
A_{1} \boldsymbol{\delta}_{1}+\left[C_{1}, 0, \ldots 0\right] \Delta+D_{1} \boldsymbol{\delta}_{J} & =\mathbf{r}_{1} \\
E \boldsymbol{\delta}_{1}+\quad a \Delta+D \boldsymbol{\delta}_{J} & =\mathbf{R} \\
E \boldsymbol{\delta}_{1}+\left[0, \ldots 0, B_{J}\right] \Delta+A_{J} \boldsymbol{\delta}_{J} & =\mathbf{r}_{J}
\end{aligned}
$$

and introduces $U$ and $V$ such that:

$$
\begin{aligned}
& a U=E, \\
& a V=D .
\end{aligned}
$$

From the definitions of $D$ and $E$ given by eqn (18), $U$ has the same form as $E$ and $V$ has the same form as $D$, so that:

$$
\begin{aligned}
& U \equiv\left(\mathbf{U}_{1}, \mathbf{U}_{2}, \mathbf{U}_{3}\right) \\
& V \equiv\left(\mathbf{V}_{1}, \mathbf{V}_{2}, \mathbf{V}_{3}\right)
\end{aligned}
$$

Substituting eqn (21) into eqn (20b), we get:

$$
a \mathbf{W}=\mathbf{R}
$$

where

$$
\mathbf{W} \equiv U \boldsymbol{\delta}_{1}+\Delta+V \boldsymbol{\delta}_{J}
$$

With eqn (24), the system (20a) and (20c) can be written as:

$$
\left|\begin{array}{ll}
\hat{A}_{1} & \hat{D}_{1} \\
\hat{E}_{J} & \hat{A}_{J}
\end{array}\right| \begin{aligned}
& \boldsymbol{\delta}_{1} \\
& \boldsymbol{\delta}_{J}
\end{aligned}|=| \begin{aligned}
& \hat{\mathbf{r}}_{1} \\
& \hat{\mathbf{r}}_{J}
\end{aligned} \mid
$$

where

$$
\begin{aligned}
\hat{A}_{1} & \equiv A_{1}-\left[C_{1}, 0, \ldots 0\right] U \\
\hat{D}_{1} & \equiv D_{1}-\left[C_{1}, 0, \ldots 0\right] V, \\
\hat{A}_{J} & \equiv A_{J}-\left[0, \ldots 0, B_{J}\right] V, \\
\hat{E}_{J} & \equiv E_{J}-\left[0, \ldots 0, B_{J}\right] U, \\
\hat{\mathbf{r}}_{1} & \equiv \mathbf{r}_{1}-\left[C_{1}, 0, \ldots 0\right] \mathbf{W}, \\
\hat{\mathbf{r}}_{J} & \equiv \mathbf{r}_{J}-\left[0, \ldots 0, B_{J}\right] \mathbf{W} .
\end{aligned}
$$

The sequence of the solution procedure is as follows. We first solve eqns (21a), (21b) and (23) to yield $U, V$ and $\mathbf{W}$, which are required in the solution of eqn (26). The resulting values of $\hat{A}_{1}, \hat{D}_{1}$, $\hat{A}_{J}, \hat{E}_{J}, \hat{\mathbf{P}}_{1}$ and $\hat{\mathbf{f}}_{J}$ are used in eqn (25), which is solved to obtain $\boldsymbol{\delta}_{1}$ and $\boldsymbol{\delta}_{J}$. Thus eqn (15) can be solved since $\Delta$ follows from eqn (24), with $\mathbf{W}, U, \delta_{1}, V$ and $\delta_{j}$ all known.

The solution of eqns (21) and (23) is obtained by the block-elimination method described in Ref. [1] and its application to eqn (23) is straightforward since $\mathbf{W}$ and $\mathbf{R}$ involve three-component vectors as in Ref. [1]. In contrast, eqn (21) involves $3 \times 3$ matrices for each of $U, V, E$ and $D$. However, with the definitions of $U$ and $V$, as given by eqn (22), and with the definitions of $E$ and $D$ given by eqns (18), eqns (21a) and (21b) can be cast in the same form as eqn (23). For example, (21a) becomes

$$
\begin{aligned}
& a \mathbf{U}_{1}=\mathbf{E}_{1} \\
& a \mathbf{U}_{2}=\mathbf{E}_{2} \\
& a \mathbf{U}_{3}=\mathbf{E}_{3},
\end{aligned}
$$




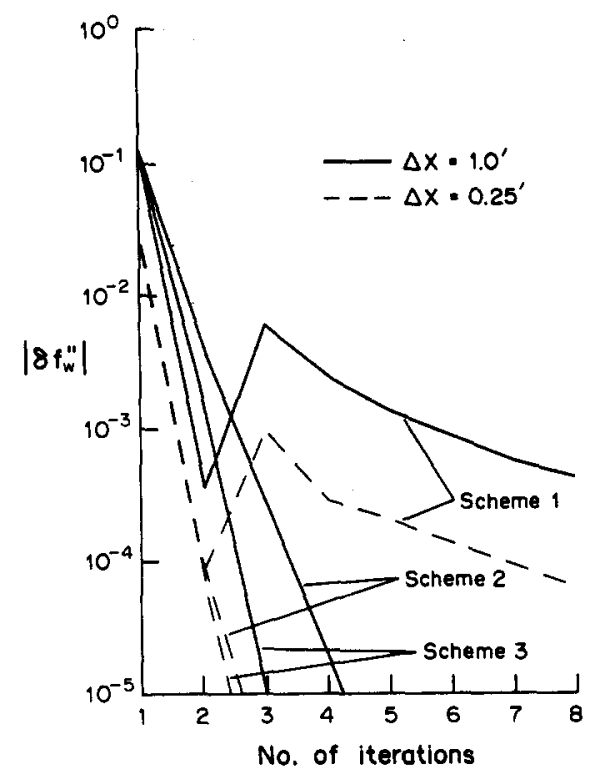

Fig. 1. Rate of convergence of three linearization schemes for two $\Delta x$-spacings.

where:

$$
\mathbf{U}_{k} \equiv\left|\begin{array}{c}
\mathbf{U}_{2, k} \\
\vdots \\
\mathbf{U}_{j, k} \\
\vdots \\
\mathbf{U}_{J-1, k}
\end{array}\right| \quad k=1,2,3 ; \quad \mathbf{U}_{j, k}=\left|\begin{array}{c}
U_{1 j k} \\
U_{2 j k} \\
U_{3 j k}
\end{array}\right| 2 \leqslant j \leqslant J-1
$$

Further details of this procedure can be found in Ref. [6].

\section{RESULTS AND DISCUSSION}

Calculations have been performed for a zero pressure gradient flow in which the flow is laminar at the leading edge of the plate with a unit Reynolds number of $10^{6} / \mathrm{ft}$; transition was specified very close to the leading edge and, as in Ref. [2], approximately eight $x$-stations were taken in the region $0<x<1^{\prime}$. Between $x=1^{\prime}$ and $x=10^{\prime}$, two different $\Delta x$-spacings corresponding to $\Delta x=0.25$ and 1 were used to study the rate of convergence of the solutions.

Figure 1 shows the results together with those of Ref. [2] at the last $x$-station corresponding to $x=10^{\prime}$. Scheme 1 corresponds to those obtained with the usual procedure in which the $b$ term in eqn (6) is assumed to be known from a previous iteration; scheme 2 corresponds to the scheme in which all the terms are linearized fully by Newton's method and solved by the procedure described in Ref. [2]: and scheme 3 corresponds to the present method which is the same as scheme 2 except that the solution procedure makes use of the full-bordering algorithm. It is evident that schemes 2 and 3 converge quadratically at a similar rate to any specified convergence criterion. In contrast, scheme 1 converges much more slowly and is limited to convergence criterion, which experience has shown to be around $1 \%$. Scheme 3 offers, therefore, the advantages of quadratic convergence expected from the application of Newton's method, together with the flexibility required to deal with complex boundary conditions and three-dimensional equations with minor modifications to the basic block-elimination method.

\section{REFERENCES}

1. T. Cebeci and P. Bradshaw, Physical and Computational Aspects of Convective Heat Transfer. Springer, New York (1984). 
2. T. Cebeci, K. C. Chang and D. P. Mack, Linearization of turbulent boundary-layer equations, A/AA J. 22, 1919.1821 (1984).

3. H. B. Keller, Practical procedures in path following near limit points. Computing Methods in Applied Sciences and Engineering (Edited by Glowinski and Lions), pp. 177 183. North Holland, Amsterdam (1982).

4. H. B. Keller, The bordering algorithm and path following near singular points of higher nullity. SIAM J. Scit. statist Comput. 4, 573-582 (1983).

5. H. B. Keller, A new difference scheme for parabolic problems. Numerical Solution of Partial-Differential Equations (Edited by J. Bramble), Vol. II. Academic Press, New York (1970).

6. H. M. Jang and T. Cebeci, A preferred approach to the linearization of turbulent boundary-layer equations. California State University, Long Beach. Aerospace Eng. Rept. AE-TR-89-1 (1989). 